\title{
AtDeg2 - a chloroplast protein with dual protease/chaperone activity
}

\author{
Przemysław Jagodzik*, Małgorzata Adamiec, Grzegorz Jackowski \\ Institute of Experimental Biology, Adam Mickiewicz University, Umultowska 89, 61-614 Poznań; Poland
}

\begin{abstract}
Chloroplast protease AtDeg2 (an ATP-independent serine endopeptidase) is cytosolically synthesized as a precursor, which is imported into the chloroplast stroma and deprived of its transit peptide. Then the mature protein undergoes routing to its functional location at the stromal side of thylakoid membrane. In its linear structure AtDeg2 molecule contains the protease domain with catalytic triad (HDS) and two PDZ domains (PDZ1 and PDZ2). In vivo AtDeg2 most probably exists as a supposedly inactive haxamer, which may change its oligomeric stage to form active 12-mer, or 24-mer. AtDeg2 has recently been demonstrated to exhibit dual protease/chaperone function. This review is focused on the current awareness with regard to AtDeg2 structure and functional significance.
\end{abstract}

Keywords: AtDeg2; chaperone; chloroplast; hexamer; protease; PDZ domain

\section{Introduction}

The results of studies performed in recent years show that regulatory protein hydrolysis, catalyzed by proteolytic enzymes occurs ubiquitously and is directly or indirectly involved in a majority (if not all) cellular processes of living organisms. It is widely believed that proteases are involved in protein quality control and protein turnover processes. The protein quality control comprises a hydrolysis of proteins, which have been damaged due to mutations or exposition of plants to stressing environmental conditions, synthesized at redundant quantity or sorted to incorrect cell compartment. Then protein turnover involves a hydrolysis of proteins, which become unnecessary in a defined ontogenetical context under comfortable environmental conditions. Aminoacids released during the course of protein turnover are reused for protein synthesis as a part of a continuous breakdown/ resynthesis of cellular components. Proteolysis is considered to regulate extensively whole-organism maintenance, structure and functions according to changes of environmental conditions and to progression of ontogenetic stages. For some proteases ATP binding and hydrolysis is necessary for their catalytic activity. All of them possess a conservative $\mathrm{AAA}^{+}$ domain which is responsible for ATP binding and hydrolysis, necessary to unfold protein substrates so that they can enter a catalytical chamber of the proteases molecule through a narrow entrance [1]. Nevertheless, some other proteases, including the Deg group, function in an ATP-independent manner, most probably because the availability of catalytic

\footnotetext{
* Corresponding author. Email: przemyslaw.jagodzik@amu.edu.pl
}

Handling Editor: Beata Zagórska-Marek center is less restricted for unfolded substrates than in the case of ATP-dependent enzymes [2].

Deg proteases comprise a very important group of proteolytic enzymes occurring in all domains of life, including Archaea, Bacteria and Eukarya [3]. Deg proteases were first discovered by studying Escherichia coli mutants unable to hydrolyze periplasmic proteins damaged under heat shock conditions $\left(>37^{\circ} \mathrm{C}\right)[4,5]$. Later it was found that the quality of $E$. coli periplasmic proteins is controlled by three Deg proteins namely DegP, DegQ and DegS whose structure and function have been precisely solved [6-8]. A unique feature of DegP protein is that it switches from protease to chaperone activity in a temperature-dependent manner. Namely at low temperatures $\left(28^{\circ} \mathrm{C}\right)$ protease activity was found to be hardly detectable and chaperone activity was high; on the other hand at elevated temperatures $\left(>28^{\circ} \mathrm{C}\right)$ the proteolytic activity rises abruptly whereas the chaperone activity is strongly diminished $[9,10]$. The chaperone activity of DegP consists in an ability to refold denatured, MalS (a native substrate) [10] and to prevent aggregation of a lysozyme (an artificial substrate) [9].

According to MEROPS database (9.10 release) count of known proteases in the model plant species Arabidopsis thaliana is 783 , representing about $3 \%$ of all proteins identified in this taxon. Sixteen genes coding for proteins orthologous to DegP, Q and S have been identified in the A. thaliana nuclear genome and designated AtDEG1-16 $[11,12]$. The genes code for proteins, which are targeted either to chloroplasts (AtDEG1, 2, 5, 7 and 8) or mitochondria (AtDEG10) or peroxisomes (AtDEG15). The localization of nine proteins encoded by remaining $A t D E G$ genes is unclear; moreover some of those nine AtDEGs may be pseudogenes [13] and two potential AtDeg proteases may 
be proteolytically inactive [12]. A majority of AtDEG genes have their orthologues in other model plant species, as Populus trichocarpa, Oryza sativa, Physcomitrella patens and Chlamydomonas reinhardtii [12].

AtDeg2 protease is an ATP-independent serine endopeptidase containing a trypsin type catalytic domain with a catalytic triad (HDS). It belongs to the DegP (E. coli) type subfamily (S1C) of the clan PA according to the MEROPS nomenclature and may be found in this database as Deg2 chloroplast peptidase (Arabidopsis thaliana). However, MEROPS database gives also the other name - AtDegP2. All AtDeg proteases were originally named as AtDegPX ones and then renamed just AtDegX [11]. It has been shown recently that AtDeg2 exhibits chaperone-like activity in vitro [14] yet no data exist with regard to how protease and chaperone activities are interlocked within a single AtDeg2 molecule.

This review focuses on current awareness with respect to structure and functions of AtDeg2 - a chloroplast protein with dual protease/chaperone activity.

\section{AtDEG2 gene}

AtDeg2 protease is encoded by nuclear AtDEG2 gene (DEGRADATION OF PERIPLASMIC PROTEINS 2) with a locus tag AT2G47940 and DDBJ/EMBL/GeneBank accession No. NC_00307, and exists at a single locus in the Arabidopsis genome. AtDEG2 gene comprises 4266 bp and is composed of 19 exons. The gene has been identified between 19617986 bp and 19622251 bp on the edge of 2 chromosome of Arabidopsis genome (DDBJ/EMBL/ GeneBank accession No. AC005309) and is "read" in opposite direction $(19622251 \rightarrow 19617986)$ AtDEG2 gene is transcribed onto mRNA (NM_130361) that contains 2278 bp and encodes a 607 aminoacids long pre-AtDeg2 protein (NP_566115.1). NCBI database indicates one more mRNA of ATDEG2 gene (NM_001125072.1) composed of 2294 bp, which is regarded as a splice variant 2 . This mRNA encodes a protein (NP_001118544.1) composed of 606 amino acids (Fig. 1). The lacking aminoacid residue (K62) is localized within an amino terminal stroma-targeting transit peptide, which is cleaved off after pre-AtDeg2 import into the plastid stoma therefore a single mature AtDeg2 isoform is found in the chloroplast.

\section{AtDeg2 protein}

\section{Localization and structure}

Hydropathy plots demonstrated mature AtDeg2 to be mostly a hydrophilic protein with no predicted transmembrane $\alpha$-helices [15]. In fact studies based on immunoblot analysis of individual chloroplast subcompartments demonstrated that AtDeg2 is associated peripherally to the stromal side of stroma thylakoid membranes (80-90\%) and non-appressed regions of grana stacks (10-20\%) [15], the observation supported by the results of LC-MS/MS analyses of proteome of subchloroplast fractions [16]. AtDeg2 is cytosolically synthesized as a precursor, which is imported into the plastid stroma and deprived of its transit peptide (positions 1-69). The mature protein (positions 70-607, i.e. 538 aminoacid residues in total) is routed to its functional location at the stromal side of thylakoid membrane [15]. Its molecular weight was assessed to be $60 \mathrm{kDa}$ [17]. According to HHPred platform (http://toolkit.tuebingen. mpg.de/hhpred/) [18] mature AtDeg2 molecule contains

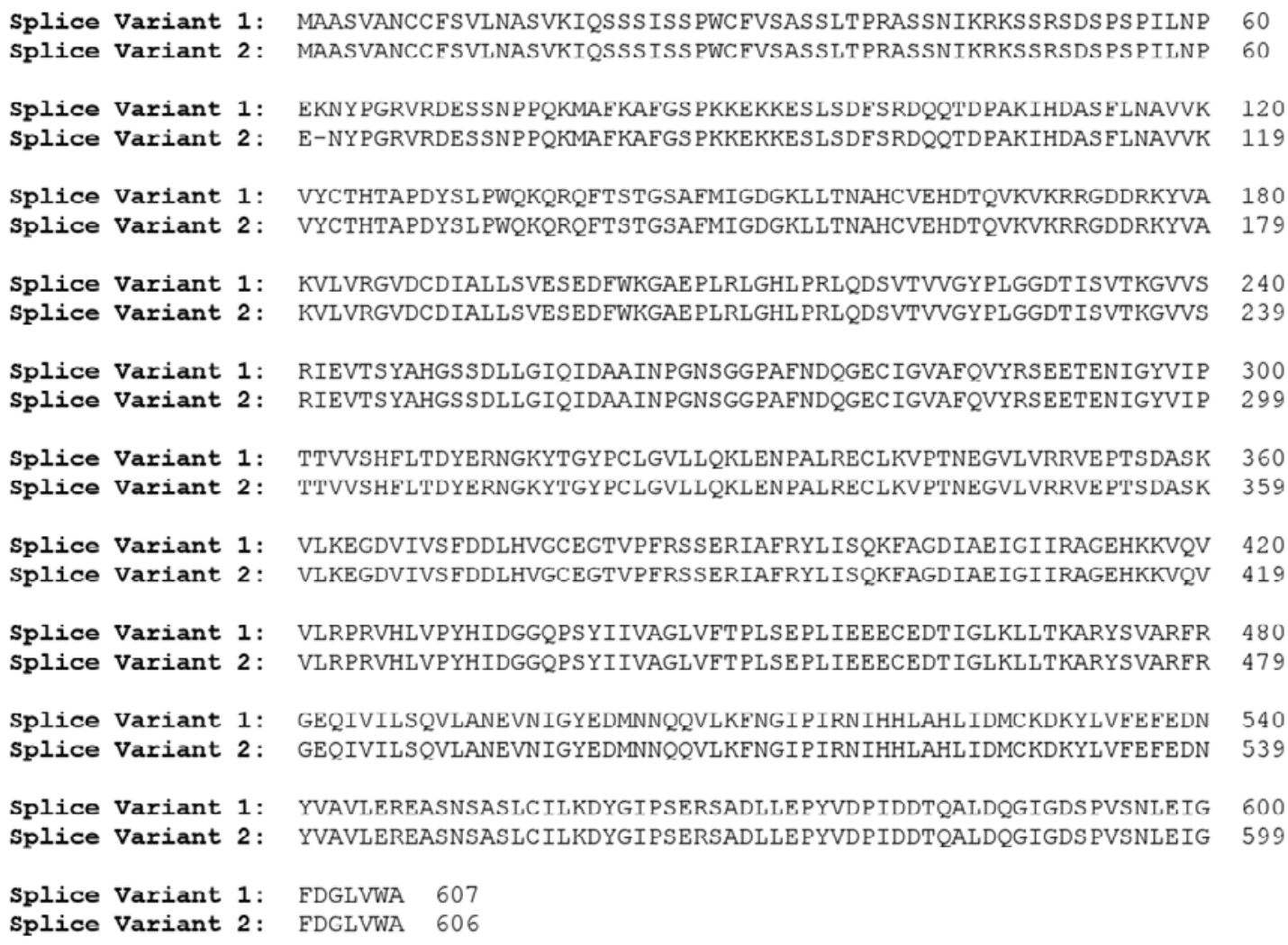

Fig. 1 Alignment of two splice variants of AtDeg2 protein. Sequences were obtained by BLAST search. 
protease domain as well PDZ1 and PDZ2 domains (positions 110-313, 314-422 and 423-577, respectively [14]). The protease domain is preceded by and PDZ2 is followed by short regions, which are lacking any conserved domains (positions 70-109 and 578-607, respectively; Fig. 2). The protease domain involves a catalytic H159 D190 S268 triad.

A great progress has been made recently with regard to understanding structural organization of AtDeg2 since recombinant version of this protein (spanning 110-577 positions) has been crystallized and the crystal structure solved based on X-ray diffraction analysis [14]. When analyzed by size exclusion chromatography AtDeg2 in solution forms supposedly inactive hexamer (a dimer of trimers) of "sealed cage" type, consisting of two trimeric rings stacked upon each other in such a way that they form an inner, catalytic chamber the entrance to which is restricted to six pores.

The formation of trimer is enabled by interactions between protease and PDZ1 domains of individual monomers (Fig. 3). In the AtDeg2 hexamer the assembly is stabilized through multiple interactions involving two interfaces formed for each monomer, consisting of the protease domain and PDZ2 (interface 1) and PDZ1 and PDZ2 (interface 2) [14]. While PDZ1 domain resembles conventional PDZ domains of bacterial Deg proteases, PDZ2 domain has a few unique features with respect to canonical PDZ domains of other Deg proteases. The most interesting feature is presence of an unusual $\beta$-strand $(\beta 21)$ comprising aminoacids $440-444$, which acts as an intramolecular internal PDZ ligand. The internal PDZ2 ligand was shown to interact with the protease domain of the same monomer by binding its LA loop (Fig. 4) thereby fixing the protease domain and making AtDeg2 monomer and hexamer rigid molecules. Apart from making AtDeg2 monomer and hexamer rigid, internal PDZ2 ligand may play a crucial role in conversion of the supposedly resting hexameric state into the enzymatically active 12-mers and 24-mers by dissociating from PDZ2 so that trimeric units may be rearranged into higher oligomeric states. The oligomerization of AtDeg2 in solution was demonstrated to be $\mathrm{pH}$-independent and to increase with the incubation time with an artificial substrate (but the hexameric state existed predominantly all the time [14]).

\section{Dual protease/chaperone activity (in vitro and in vivo)}

Recombinant AtDeg2 catalyzed in vitro hydrolysis of various artificial protein substrates as gelatin [15], fluorescencelabelled casein [19] and $\beta$-casein [14] thereby demonstrating to be bona fide proteolytic enzyme. AtDeg2 proteolityc activity in vitro was found to be regulated in redox- and $\mathrm{pH}$-dependent manner $[14,19]$ and it was suggested that cysteine residues found in AtDeg2 molecule may trigger

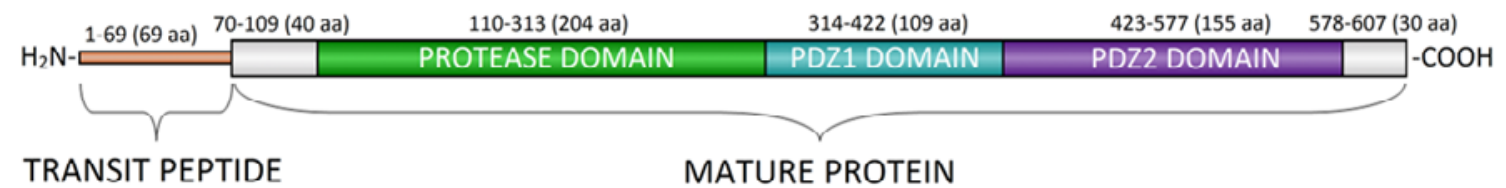

Fig. 2 The distribution of conservative domains in a linear structure of the precursor of AtDeg2 protein. The distribution of the domains is shown after [14] and the determination of processing site was performed using TARGETP 1.1 Server (http://www.cbs.dtu.dk/services/ TargetP/). aa - aminoacids.
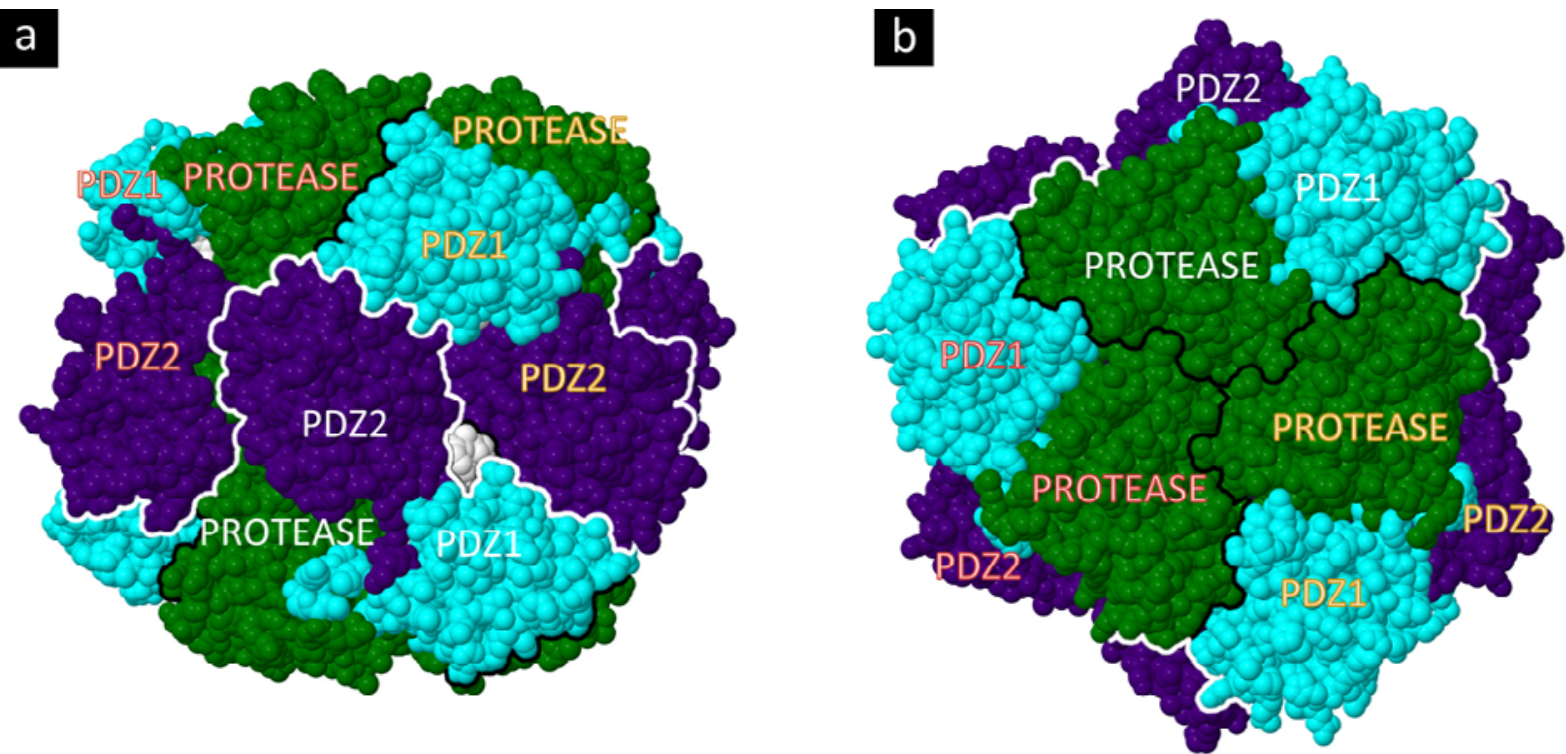

Fig. 3 Space fill representation of side (a) and top (b) views of structure of AtDeg2 hexamer (MMDB: 103069; PDB ID: 4FLN). Appropriate domains are distinguished by different colours (green - protease domain; cyan - PDZ1 domain; indigo - PDZ2 domain; grey - co-crystalized peptide). Monomers are divided by black line and trimers by white line. View in Jmol (Jmol: an open-source Java viewer for chemical structures in 3D - http://www.jmol.org/). 
a

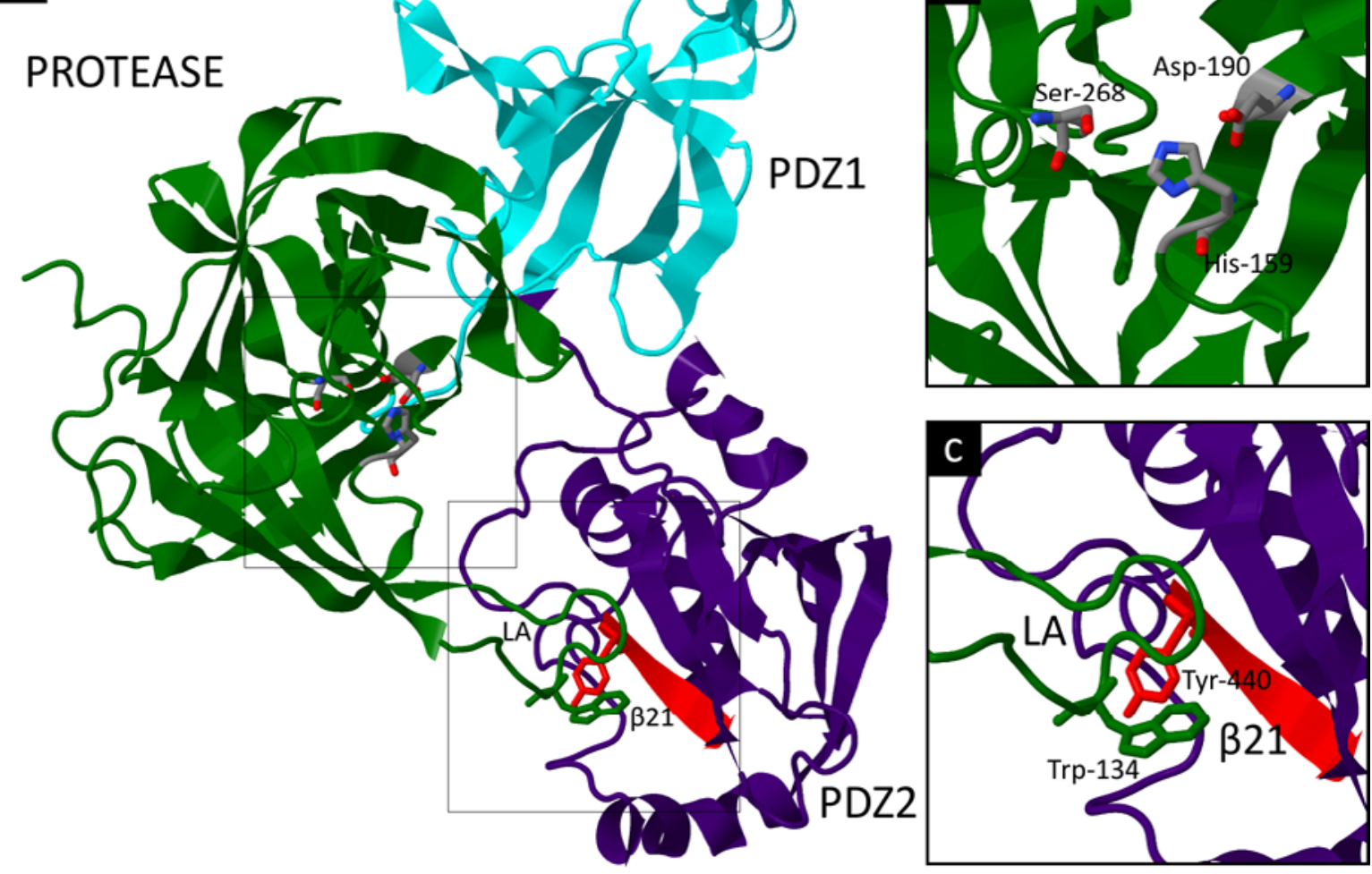

Fig. 4 Cartoon representation of structure of AtDeg2 monomer (a), catalytic triad (b) and PDZ2 ligand red colored (c). Appropriate domains are distinguished by different colors (green - protease domain; cyan - PDZ1 domain; indigo - PDZ2 domain). The catalytic triad and amino acids participating in LA loop binding are shown as a stick model (MMDB ID: 103069; PDB ID: 4FLN). View in Jmol (Jmol: an open-source Java viewer for chemical structures in 3D - http://www.jmol.org/).

redox-dependent conformational changes of the molecule leading to modifications of proteolytic activity observed in various redox surroundings [19]. However, extremely little is known about the identity of native substrates for AtDeg2 under stressing or non-stressing conditions in vivo. It was revealed in this respect that AtDeg2 might be involved in vivo in degradation of photodamaged PsbA protein by catalyzing the primary cleavage of stroma-exposed DE loop [15]. Yet, the role of this protease in degradation of PsbA was questioned since later it was shown that photodamaged PsbA protein had been effectively degraded in A. thaliana mutant lacking AtDeg2 [20]. The protease has recently been demonstrated to take part in protection against photoinhibition [17] again indicating possible involvement of this protease in removing photodamaged D1 protein in vivo. The only more straightforward data concerning identity of native substrates for AtDeg2 come from our laboratory - an apoprotein Lhcb6 has recently been found to be a target for short stress (3 h)-related degradation catalyzed by AtDeg2, as judged by an inability of $A$. thaliana mutants devoid of AtDeg2 to cleave Lhcb6 in leaves exposed to various stresses (elevated irradiance, heat, high salt and wounding). Most probably Lhcb6 apoprotein experiences an oxidative damage as result of short stresses in a manner that marks this protein for AtDeg2-dependent recognition and hydrolysis [17].

It was thought for several years that in contrast to what is observed with respect to DegP, chloroplast-targeted AtDegs function uniquely as proteases, i.e. are not able to switch reversibly from protease to chaperone and it was only in last four years that intriguing observations have been published demonstrating that AtDeg2 (and AtDeg1) may function as chaperones as well. Namely recombinant AtDeg1 was demonstrated to stimulate refolding of unfolded version of MalS protein [21] and recombinant AtDeg2 inhibited aggregation of denatured lysozyme in vitro [14]. However, both AtDeg1 and AtDeg2 exhibited dual activity in a wide range of temperatures in contrast to temperature-dependent shifting between protease and chaperone functions demonstrated for DegP. It was found that chaperone activity of AtDeg1 is confined to catalytic triad of protease domain [21] yet no data exist concerning a localization of chaperone activity in a linear structure of AtDeg2 molecule [14].

\section{Functional importance}

AtDEG2 is expressed almost ubiquitously, practically through all ontogenetic stages of the model plant species [22,23]. When AthaMap (http://www.athamap.de/) [24] was used to search for transcription binding elements within AtDEG2 promoter predicted by AGRIS (http://arabidopsis. med.ohio-state.edu/) [25] eight well known and highly conserved cis-regulatory elements have been identified, specific for ARR-B, GATA, CAMTA, LFY, NAC and AP2/EREBP families of transcription factors. Namely, AtDEG2 promoter contains two individual ARR-B recognition sequences, known as ARR1 and ARR2 and identified as key transcription activators in cytokinin downstream signaling pathway 
[26]. Two potential targets for GATA transcription factors were also detected. One of the motifs designated GATA-1 was found to be involved in light regulation of nuclear genes expression [27] whereas the second one (described as ZML2) was identified as playing a crucial role in the cryptochrome1-dependent response to excess light [28]. In addition, three identical cis-regulatory elements recognizing LFY and single cis-regulatory elements specific for CAMTA3 (CAMTA family), NAP (NAC family) and RAP2.2 (AP2/ EREBP family) transcription factors have been detected. LFY transcription factor is thought to play significant role in photoperiodic-dependent gene expression regulatory pathways [29], CAMTA3 in turn is considered to be involved in responses to biotic stresses [30], cold and freezing tolerance [31] as well as in regulation of ethylene-induced senescence [32]. NAP plays a key role in leaf senescence by participation in regulatory pathway which controls stomatal movement and water loss during leaf senescence [33] and the RAP2.2 was found to be involved in transactivation of two genes coding for enzymes engaged in carotenogenesis [34]. It is not easy to imagine how AtDEG2 transactivation, exerted by interactions between the above mentioned transcription factors and cis-regulatory motifs within AtDEG2 promoter may influence growth and development events since reliable data regarding the role of AtDeg2 in controlling the course of growth and developmental processes at the whole plant level are very scarce. It was demonstrated by us that under non-stressing conditions AtDeg2 is involved in regulation of both morphology and chloroplast life cycle of juvenile rosette leaves - at the moment when first flower was opened the area of juvenile leaves of mutants devoid of AtDeg2 was reduced significantly with respect to those of wild type plants (the alteration was found to be photoperiod independent). At the same moment chloroplasts of the wild plants' juvenile leaves showed signs of entering a senescence phase (e.g. the presence of numerous, large plastoglobules and periodic undulations of thylakoids parallel to those of the envelope - Fig. 5) which chloroplasts of mutants devoid of AtDeg2 appear not to do [17]. AtDEG2 transactivation phenomena may mediate regulatory events triggered in response to exposure of $A$. thaliana plants to abiotic stress conditions as well, as judged by the fact that AtDeg2 mRNA accumulation is remarkably downregulated in response to short-term $(2 \mathrm{~h})$ exposure of detached mature leaves to a variety of abiotic stress conditions including heat, high salt

\section{Acknowledgments}

The project was supported by grant from Polish National Science Center based on decision DEC-2013/09/B/NZ3/00449.

\section{Authors' contributions}

The following declarations about authors' contributions to the research have been made: worked out the idea of the review: GJ, PJ; wrote the text: GJ, PJ, MA; performed the analyses with AGRIS and AthaMap tools: MA.

\section{References}

1. Narberhaus F, Obrist M, Führer F, Langklotz S. Degradation of cytoplasmic substrates by FtsH, a membrane-anchored protease with many talents. Res Microbiol. 2009;160(9):652-659. http://dx.doi. org/10.1016/j.resmic.2009.08.011 or desiccation [15] and upregulated in response to short term exposure $(2.5 \mathrm{~h})$ to elevated irradiance [35]. On the contrary long-term exposure $(5 \mathrm{~h})$ to elevated irradiance was found to be accompanied by a reduction in AtDeg2 transcript level [36]. Usually only a weak correlation exists between the accumulation of numerous chloroplast protease transcript and their proteins including AtDeg2 [15,37] thereby complex AtDeg2 regulatory mechanisms including transcriptional/ translational as well as posttranscriptional phenomena [38] have to be triggered in response to the action of exogenous stressing factors as well as in response to progression of ontogenetic phases.
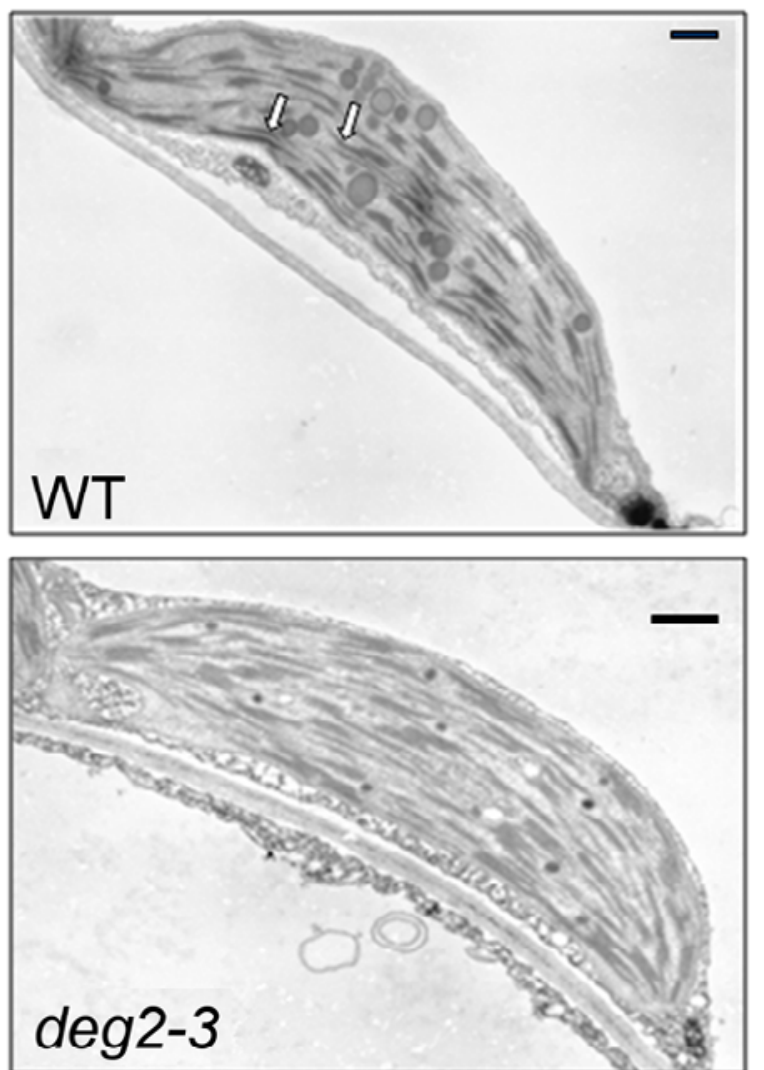

Fig. 5 Transmission electron microscopy of chloroplast of juvenile leaves of Arabidopsis thaliana wild type plants (WT) and mutants devoid of AtDeg2 protease (deg2-3) [17]. Micrographs show chloroplasts of mesophyll cells of plants which reached the moment when the first flower opened. [17]. Arrows point to the undulations of thylakoids of chloroplast of WT plants; their appearance marks the onset of early senescence. Scale bar: $500 \mathrm{~nm}$.
2. Kley J, Schmidt B, Boyanov B, Stolt-Bergner PC, Kirk R, Ehrmann $\mathrm{M}$, et al. Structural adaptation of the plant protease Deg1 to repair photosystem II during light exposure. Nat Struct Mol Biol. 2011;18(6):728-731. http://dx.doi.org/10.1038/nsmb.2055

3. Clausen T, Southan C, Ehrmann M. The HtrA family of proteases: implications for protein composition and cell fate. Mol Cell. 2002;10(3):443-455. http://dx.doi.org/10.1016/S1097-2765(02)00658-5

4. Lipinska B, Sharma S, Georgopoulos C. Sequence analysis and regulation of the htrA gene of Escherichia coli: a $\sigma^{32}$-independent mechanism of heat-inducible transcription. Nucleic Acids Res. 1988;16(21):10053-10067. http://dx.doi.org/10.1093/nar/16.21.10053

5. Strauch KL, Beckwith J. An Escherichia coli mutation preventing degradation of abnormal periplasmic proteins. Proc Natl Acad Sci USA. 1988;85(5):1576-1580. http://dx.doi.org/10.1073/pnas.85.5.1576 
6. Wilken C, Kitzing K, Kurzbauer R, Ehrmann M, Clausen T. Crystal structure of the DegS stress sensor: how a PDZ domain recognizes misfolded protein and activates a protease. Cell. 2004;117(4):483-494. http://dx.doi.org/10.1016/S0092-8674(04)00454-4

7. Jiang J, Zhang X, Chen Y, Wu Y, Zhou ZH, Chang Z, et al. Activation of DegP chaperone-protease via formation of large cagelike oligomers upon binding to substrate proteins. Proc Natl Acad Sci USA. 2008;105(33):11939-11944. http://dx.doi.org/10.1073/ pnas. 0805464105

8. Bai XC, Pan XJ, Wang XJ, Ye YY, Chang LF, Leng D, et al. Characterization of the structure and function of Escherichia coli DegQ as a representative of the DegQ-like proteases of bacterial HtrA family proteins. Structure. 2011;19(9):1328-1337. http://dx.doi.org/10.1016/j. str.2011.06.013

9. Skórko-Glonek J, Krzewski K, Lipińska B, Bertoli E, Tanfani F. Comparison of the structure of wild-type HtrA heat shock protease and mutant HtrA proteins. A Fourier transform infrared spectroscopic study. J Biol Chem. 1995;270(19):11140-11146. http://dx.doi. org/10.1074/jbc.271.52.33502

10. Spiess C, Beil A, Ehrmann M. A temperature-dependent switch from chaperone to protease in a widely conserved heat shock protein. Cell. 1999;97(3):339-347. http://dx.doi.org/10.1016/S0092-8674(00)80743-6

11. Huesgen PF, Schuhmann H, Adamska I. The family of Deg proteases in cyanobacteria and chloroplasts of higher plants. Physiol Plant. 2005;123(4):413-420. http://dx.doi.org/10.1111/j.1399 $-3054.2005 .00458 . x$

12. Schuhmann H, Huesgen PF, Adamska I. The family of Deg/HtrA proteases in plants. BMC Plant Biol. 2012;12(1):52. http://dx.doi. org/10.1186/1471-2229-12-52

13. Schuhmann H, Adamska I. Deg proteases and their role in protein quality control and processing in different subcellular compartments of the plant cell. Physiol Plant. 2012;145(1):224-234. http://dx.doi. org/10.1111/j.1399-3054.2011.01533.x

14. Sun R, Fan H, Gao F, Lin Y, Zhang L, Gong W, et al. Crystal structure of Arabidopsis Deg2 protein reveals an internal PDZ ligand locking the hexameric resting state. J Biol Chem. 2012;287(44):37564-37569. http://dx.doi.org/10.1074/jbc.M112.394585

15. Haussuhl K, Andersson B, Adamska I. A chloroplast DegP2 protease performs the primary cleavage of the photodamaged D1 protein in plant photosystem II. EMBO J. 2001;20(4):713-722. http://dx.doi. org/10.1093/emboj/20.4.713

16. Ferro M, Brugiere S, Salvi D, Seigneurin-Berny D, Court M, Moyet L, et al. AT_CHLORO, a comprehensive chloroplast proteome database with subplastidial localization and curated information on envelope proteins. Mol Cell Proteomics. 2010;9(6):1063-1084. http://dx.doi. org/10.1074/mcp.M900325-MCP200

17. Luciński R, Misztal L, Samardakiewicz S, Jackowski G. The thylakoid protease Deg2 is involved in stress-related degradation of the photosystem II light-harvesting protein Lhcb6 in Arabidopsis thaliana. New Phytol. 2011;192(1):74-86. http://dx.doi. org/10.1111/j.1469-8137.2011.03782.x

18. Soding J, Biegert A, Lupas AN. The HHpred interactive server for protein homology detection and structure prediction. Nucleic Acids Res. 2005;33:W244-W248. http://dx.doi.org/10.1093/nar/gki408

19. Ströher E, Dietz KJ. The dynamic thiol-disulphide redox proteome of the Arabidopsis thaliana chloroplast as revealed by differential electrophoretic mobility. Physiol Plant. 2008;133(3):566-583. http:// dx.doi.org/10.1111/j.1399-3054.2008.01103.x

20. Huesgen PF, Schuhmann H, Adamska I. Photodamaged D1 protein is degraded in Arabidopsis mutants lacking the Deg2 protease. FEBS Lett. 2006;580(30):6929-6932. http://dx.doi.org/10.1016/j. febslet.2006.11.058

21. Sun X, Ouyang M, Guo J, Ma J, Lu C, Adam Z, et al. The thylakoid protease Deg1 is involved in photosystem-II assembly in Arabidopsis thaliana: chaperone function of Deg1. Plant J. 2010;62(2):240-249. http://dx.doi.org/10.1111/j.1365-313X.2010.04140.x

22. Schmid M, Davison TS, Henz SR, Pape UJ, Demar M, Vingron M, et al. A gene expression map of Arabidopsis thaliana development. Nat Genet. 2005;37(5):501-506. http://dx.doi.org/10.1038/ng1543
23. Nakabayashi K, Okamoto M, Koshiba T, Kamiya Y, Nambara E. Genome-wide profiling of stored mRNA in Arabidopsis thaliana seed germination: epigenetic and genetic regulation of transcription in seed: molecular profiling in Arabidopsis seed. Plant J. 2005;41(5):697-709. http://dx.doi.org/10.1111/j.1365-313X.2005.02337.x

24. Yilmaz A, Mejia-Guerra MK, Kurz K, Liang X, Welch L, Grotewold E. AGRIS: the Arabidopsis gene regulatory information server, an update. Nucleic Acids Res. 2011;39:D1118-D1122. http://dx.doi. org/10.1093/nar/gkq1120

25. Hehl R, Bülow L. AthaMap web tools for the analysis of transcriptional and posttranscriptional regulation of gene expression in Arabidopsis thaliana. In: Staiger D, editor. Plant circadian networks. New York, NY: Springer New York; 2014. p. 139-156. (Methods in molecular biology; vol 1158). http://dx.doi.org/10.1007/978-1-4939-0700-7_9

26. Hwang I, Sheen J. Two-component circuitry in Arabidopsis cytokinin signal transduction. Nature. 2001;413(6854):383-389. http://dx.doi. org/10.1038/35096500

27. Jeong MJ, Shih MC. Interaction of a GATA factor with cis-acting elements involved in light regulation of nuclear genes encoding chloroplast glyceraldehyde-3-phosphate dehydrogenase in Arabidopsis. Biochem Biophys Res Commun. 2003;300(2):555-562. http://dx.doi. org/10.1016/S0006-291X(02)02892-9

28. Shaikhali J, de Dios Barajas-Lopez J, Otvos K, Kremnev D, Garcia AS, Srivastava $\mathrm{V}$, et al. The CRYPTOCHROME1-dependent response to excess light is mediated through the transcriptional activators ZINC FINGER PROTEIN EXPRESSED IN INFLORESCENCE MERISTEM LIKE1 and ZML2 in Arabidopsis. Plant Cell. 2012;24(7):3009-3025. http://dx.doi.org/10.1105/tpc.112.100099

29. Benlloch R, Kim MC, Sayou C, Thévenon E, Parcy F, Nilsson O. Integrating long-day flowering signals: a LEAFY binding site is essential for proper photoperiodic activation of APETALA1. Plant J. 2011;67(6):1094-1102. http://dx.doi. org/10.1111/j.1365-313X.2011.04660.x

30. Galon Y, Nave R, Boyce JM, Nachmias D, Knight MR, Fromm H. Calmodulin-binding transcription activator (CAMTA) 3 mediates biotic defense responses in Arabidopsis. FEBS Lett. 2008;582(6):943-948. http://dx.doi.org/10.1016/j.febslet.2008.02.037

31. Doherty CJ, van Buskirk HA, Myers SJ, Thomashow MF. Roles for Arabidopsis CAMTA transcription factors in cold-regulated gene expression and freezing tolerance. Plant Cell. 2009;21(3):972-984. http://dx.doi.org/10.1105/tpc.108.063958

32. Nie H, Zhao C, Wu G, Wu Y, Chen Y, Tang D. SR1, a calmodulin-binding transcription factor, modulates plant defense and ethylene-induced senescence by directly regulating NDR1 and EIN3. Plant Physiol. 2012;158(4):1847-1859. http://dx.doi.org/10.1104/pp.111.192310

33. Zhang K, Gan SS. An abscisic acid-AtNAP transcription factor-SAG113 protein phosphatase $2 \mathrm{C}$ regulatory chain for controlling dehydration in senescing Arabidopsis leaves. Plant Physiol. 2012;158(2):961-969. http://dx.doi.org/10.1104/pp.111.190876

34. Welsch R, Maass D, Voegel T, DellaPenna D, Beyer P. Transcription factor RAP2.2 and its interacting partner SINAT2: stable elements in the carotenogenesis of Arabidopsis leaves. Plant Physiol. 2007;145(3):1073-1085. http://dx.doi.org/10.1104/pp.107.104828

35. Sinvany-Villalobo G, Davydov O, Ben-Ari G, Zaltsman A, Raskind A, Adam Z. Expression in multigene families. Analysis of chloroplast and mitochondrial proteases. Plant Physiol. 2004;135(3):1336-1345. http://dx.doi.org/10.1104/pp.104.043299

36. Adamiec M, Luciński R, Jackowski G. The irradiance dependent transcriptional regulation of AtCLPB3 expression. Plant Sci. 2011;181(4):449-456. http://dx.doi.org/10.1016/j.plantsci.2011.07.004

37. Zheng B, Halperin T, Hruskova-Heidingsfeldova O, Adam Z, Clarke AK. Characterization of chloroplast Clp proteins in Arabidopsis: localization, tissue specificity and stress responses. Physiol Plant. 2002;114(1):92-101. http://dx.doi. org/10.1034/j.1399-3054.2002.1140113.x

38. Żelisko A, Jackowski G. Senescence-dependent degradation of Lhcb3 is mediated by a thylakoid membrane-bound protease. J Plant Physiol. 2004;161(10):1157-1170. http://dx.doi.org/10.1016/j.jplph.2004.01.006 